1 Hacettepe Journal of Mathematics and Statistics

$\bigcap$ Volume 47 (3) (2018), 567-578

\title{
New upper bounds of Ostrowski type integral inequalities utilizing Taylor expansion
}

\author{
Hüseyin Budak*, Fuat Usta ${ }^{\dagger \ddagger}$ and Mehmet Zeki Sarıkaya ${ }^{\S}$
}

\begin{abstract}
In this paper, we have been introduced and tested some significant new bounds of Ostrowski type integral inequalities. In accordance with this purpose we have taken advantageous of the Taylor expansion for functions. Some numerical experiments have been given to show the applicability and accuracy of the proposed method.
\end{abstract}

Keywords: Ostrowski inequality, Taylor expansion.

2000 AMS Classification: 26D15, 26B25, 26D10.

Received: 14.02.2017 Accepted : 18.04.2017 Doi : 10.15672/HJMS.2017.472

\section{Introduction and Preliminaries}

In 1938, Ostrowski proved the following integral inequality. The inequality of Ostrowski [12] gives us an estimate for the deviation of the values of a smooth function from its mean value. More precisely, if $f:[a, b] \rightarrow \mathbb{R}$ is a differentiable function with bounded derivative, then

$$
\left|f(x)-\frac{1}{b-a} \int_{a}^{b} f(t) d t\right| \leq\left[\frac{1}{4}+\frac{\left(x-\frac{a+b}{2}\right)^{2}}{(b-a)^{2}}\right](b-a)\left\|f^{\prime}\right\|_{\infty}
$$

for every $x \in[a, b]$. Moreover the constant $1 / 4$ is the best possible.

Recently, several generalisations of the Ostrowski integral inequality for mappings of bounded variation and for Lipschitzian, monotonic, absolutely continuous and n-times

*Department of Mathematics, Faculty of Science and Arts, Düzce University, Düzce-Turkey, Email: hsyn.budak@gmail.com

$\dagger$ Department of Mathematics, Faculty of Science and Arts, Düzce University, Düzce-Turkey, Email: fuatusta@duzce.edu.tr

$\ddagger_{\text {Corresponding Author. }}$

$\S$ Department of Mathematics, Faculty of Science and Arts, Düzce University, Düzce-Turkey, Email: sarikayamz@gmail.com 
differentiable mappings with error estimates for some special means and for some numerical quadrature rules are considered by many authors. For recent results and generalizations concerning Ostrowski's inequality see [2]-[11], [13]-[16] and the references therein.

1.1. Theorem. [1]Let $f:[a, b] \rightarrow \mathbb{R}$ and let $n$ be a positive integer. If $f$ is such that $f^{(n)}$ is absolutely continuous on $[a, b], x_{0} \in(a, b)$, then for all $x \in(a, b)$ we have

$$
f(x)=T_{n}\left(f ; x_{0}, x\right)+R_{n}\left(f ; x_{0}, x\right)
$$

where $T_{n}\left(f ; x_{0}, \cdot\right)$ is Taylor's polynomial of degree $n$, i.e.,

$$
T_{n}\left(f ; x_{0}, x\right)=\sum_{k=0}^{n} \frac{f^{(k)}\left(x_{0}\right)\left(x-x_{0}\right)^{k}}{k !}
$$

(note that $f^{(0)}=f$ and $\left.0 !=1\right)$, and the remainder can be given by

$$
R_{n}\left(f ; x_{0}, x\right)=\frac{1}{n !} \int_{x_{0}}^{x}(x-t)^{n} f^{(n+1)}(t) d t .
$$

\section{Main Findings \& Cumulative Results}

2.1. Theorem. Let Let $f:[a, b] \rightarrow \mathbb{R}$ be a twice continuously differentiable mapping on $(a, b)$ with $f^{\prime \prime} \in L^{p}(a, b), 1 \leqq p<\infty$, we have

$$
\left|\frac{1}{b-a} \int_{a}^{b} f(t) d t-\frac{(x-a) f(a)+(b-x) f(b)}{2(b-a)}-\frac{1}{2} f(x)\right| \leq A(q, x)\left\|f^{\prime \prime}\right\|_{p}
$$

for all $x \in[a, b]$, where

$$
A(x, q)=\frac{1}{2(b-a)}\left[\left(\frac{(x-a)^{2 q+1}+(b-x)^{2 q+1}}{2 q+1}\right)^{\frac{1}{q}}+2(b-a)\left(\frac{(x-a)^{q+1}}{q+1}\right)^{\frac{1}{q}}\right]
$$

and $\frac{1}{p}+\frac{1}{q}=1$.

Proof. Define the mapping $F:[a, b] \rightarrow \mathbb{R}$ given by

$$
F(x)=\int_{a}^{x} f(t) d t
$$

If we choose $f(x)=F(x)$ and $x_{0}=a$ up to third term in Theorem 1.1, we get

$$
F(x)=F(a)+(x-a) F^{\prime}(a)+\frac{1}{2}(x-a)^{2} F^{\prime \prime}(a)+\frac{1}{2} \int_{a}^{x}(x-t)^{2} F^{\prime \prime \prime}(t) d t
$$

which yields

$$
F(x)=(x-a) f(a)+\frac{1}{2}(x-a)^{2} f^{\prime}(a)+\frac{1}{2} \int_{a}^{x}(x-t)^{2} f^{\prime \prime}(t) d t .
$$

Similarly, for $f(x)=F(x)$ and $x_{0}=b$ in Theorem 1.1, we have

$$
F(x)=F(b)+(x-b) F^{\prime}(b)+\frac{1}{2}(x-b)^{2} F^{\prime \prime}(b)+\frac{1}{2} \int_{b}^{x}(x-t)^{2} F^{\prime \prime \prime}(t) d t
$$


which yields

$$
F(x)=\int_{a}^{b} f(t) d t+(x-b) f(b)+\frac{1}{2}(x-b)^{2} f^{\prime}(b)+\frac{1}{2} \int_{b}^{x}(x-t)^{2} f^{\prime \prime}(t) d t .
$$

If we subtract equality (2.2) from equality (2.1), we have the following integral equality:

$$
\begin{aligned}
\int_{a}^{b} f(t) d t & =(x-a) f(a)+(b-x) f(b)+\frac{1}{2}(x-a)^{2} f^{\prime}(a)-\frac{1}{2}(x-b)^{2} f^{\prime}(b) \\
& +\frac{1}{2} \int_{a}^{b}(x-t)^{2} f^{\prime \prime}(t) d t .
\end{aligned}
$$

Then, we have

$$
\begin{aligned}
& \frac{1}{b-a} \int_{a}^{b} f(t) d t-\frac{(x-a) f(a)+(b-x) f(b)}{b-a} \\
= & \frac{1}{2(b-a)}\left[(x-a)^{2} f^{\prime}(a)-(x-b)^{2} f^{\prime}(b)+\int_{a}^{b}(x-t)^{2} f^{\prime \prime}(t) d t\right] .
\end{aligned}
$$

On the other hand, using Theorem 1.1 again, for $x_{0}=a$ and $x_{0}=b$ up to second term, we deduce that

$$
f(x)=f(a)+(x-a) f^{\prime}(a)+\int_{a}^{x}(x-t) f^{\prime \prime}(t) d t
$$

and

$$
f(x)=f(b)+(x-b) f^{\prime}(b)+\int_{b}^{x}(x-t) f^{\prime \prime}(t) d t .
$$

Multiplying equality (2.2) and equality $(2.1)$ by $-(x-a)$ and $(x-b)$, respectively, and then adding the resulting equalities, we find that

$$
\begin{aligned}
(a-b) f(x) & =-(x-a) f(a)-(b-x) f(b) \\
& -(x-a)^{2} f^{\prime}(a)+(b-x)^{2} f^{\prime}(b) \\
& -(x-a) \int_{a}^{x}(x-t) f^{\prime \prime}(t) d t-(b-x) \int_{b}^{x}(x-t) f^{\prime \prime}(t) d t .
\end{aligned}
$$

Dividing both sides of equality $(2.7)$ by $2(b-a)$, we obtain

$$
\begin{aligned}
& \frac{(x-a) f(a)+(b-x) f(b)}{2(b-a)}-\frac{1}{2} f(x) \\
= & -\frac{1}{2(b-a)}\left[(x-a)^{2} f^{\prime}(a)-(b-x)^{2} f^{\prime}(b)\right. \\
+ & \left.(x-a) \int_{a}^{x}(x-t) f^{\prime \prime}(t) d t+(b-x) \int_{b}^{x}(x-t) f^{\prime \prime}(t) d t\right] .
\end{aligned}
$$


Combining (2.3) and (2.7), we deduce that

$$
\begin{aligned}
& \frac{1}{b-a} \int_{a}^{b} f(t) d t-\frac{(x-a) f(a)+(b-x) f(b)}{2(b-a)}-\frac{1}{2} f(x) \\
= & \frac{1}{2(b-a)}\left[\int_{a}^{b}(x-t)^{2} f^{\prime \prime}(t) d t-(x-a) \int_{a}^{x}(x-t) f^{\prime \prime}(t) d t\right. \\
- & \left.(b-x) \int_{b}^{x}(x-t) f^{\prime \prime}(t) d t\right] .
\end{aligned}
$$

To end the proof, it remains to find an upper bound of equality (2.8). Therefore using the properties of modulus, we have

$$
\begin{aligned}
& \left|\frac{1}{b-a} \int_{a}^{b} f(t) d t-\frac{(x-a) f(a)+(b-x) f(b)}{2(b-a)}-\frac{1}{2} f(x)\right| \\
\leq & \frac{1}{2(b-a)}\left[\left|\int_{a}^{b}(x-t)^{2} f^{\prime \prime}(t) d t\right|+\left|(x-a) \int_{a}^{x}(x-t) f^{\prime \prime}(t) d t\right|\right. \\
+ & \left.\left|(b-x) \int_{b}^{x}(x-t) f^{\prime \prime}(t) d t\right|\right] .
\end{aligned}
$$

Then, by the well-known Hölder inequality, one finds that

$$
\begin{aligned}
\left|\int_{a}^{b}(x-t)^{2} f^{\prime \prime}(t) d t\right| & \leq\left\|f^{\prime \prime}\right\|_{p}\left(\int_{a}^{b}(x-t)^{2 q} d t\right)^{\frac{1}{q}} \\
& =\left\|f^{\prime \prime}\right\|_{p}\left(\frac{(x-a)^{2 q+1}+(b-x)^{2 q+1}}{2 q+1}\right)^{\frac{1}{q}} \\
\left|\int_{a}^{x}(x-t) f^{\prime \prime}(t) d t\right| & \leq\left\|f^{\prime \prime}\right\|_{p}\left(\int_{a}^{x}(x-t)^{q} d t\right)^{\frac{1}{q}} \\
& =\left\|f^{\prime \prime}\right\|_{p}\left(\frac{(x-a)^{q+1}}{q+1}\right)^{\frac{1}{q}} .
\end{aligned}
$$

If we substitute (2.10) and (2.11) into (2.9), we have

$$
\begin{aligned}
& \left|\frac{1}{b-a} \int_{a}^{b} f(t) d t-\frac{(x-a) f(a)+(b-x) f(b)}{2(b-a)}-\frac{1}{2} f(x)\right| \\
\leq & \frac{\left\|f^{\prime \prime}\right\|_{p}}{2(b-a)}\left[\left(\frac{(x-a)^{2 q+1}+(b-x)^{2 q+1}}{2 q+1}\right)^{\frac{1}{q}}+2(b-a)\left(\frac{(x-a)^{q+1}}{q+1}\right)^{\frac{1}{q}}\right] .
\end{aligned}
$$

The theorem is completely proved.

2.2. Corollary. Under the assumptions of Theorem 2.1, 
1) if we choose $x=a$, then we have the following trapezoid type inequality

$$
\left|\frac{1}{b-a} \int_{a}^{b} f(t) d t-\frac{f(a)+f(b)}{2}\right| \leq \frac{(b-a)^{1+\frac{1}{q}}}{2(2 q+1)^{\frac{1}{q}}}\left\|f^{\prime \prime}\right\|_{p},
$$

2) if we choose $x=b$, then we have the following trapezoid type inequality

$$
\left|\frac{1}{b-a} \int_{a}^{b} f(t) d t-\frac{f(a)+f(b)}{2}\right| \leq\left[\frac{1}{2(2 q+1)^{\frac{1}{q}}}+\frac{1}{(q+1)^{\frac{1}{q}}}\right](b-a)^{1+\frac{1}{q}}\left\|f^{\prime \prime}\right\|_{p},
$$

3) if we choose $x=\frac{a+b}{2}$, then we have the following Bullen type inequality

$$
\begin{aligned}
& \left|\frac{1}{b-a} \int_{a}^{b} f(t) d t-\frac{1}{2}\left[\frac{f(a)+f(b)}{2}+f\left(\frac{a+b}{2}\right)\right]\right| \\
\leq & \frac{(b-a)^{1+\frac{1}{q}}}{2}\left[\frac{1}{4(2 q+1)^{\frac{1}{q}}}+\frac{1}{[2(q+1)]^{\frac{1}{q}}}\right]\left\|f^{\prime \prime}\right\|_{p} .
\end{aligned}
$$

2.3. Theorem. Let $f:[a, b] \rightarrow \mathbb{R}$ be a twice continuously differentiable mapping on $(a, b)$ with $f^{\prime \prime} \in L^{p}(a, b), 1 \leqq p<\infty$, we have

$$
\left|f(x)-\frac{1}{b-a} \int_{a}^{b} f(t) d t-\left(x-\frac{a+b}{2}\right) f^{\prime}(x)\right| \leq B(x, q)\left\|f^{\prime \prime}\right\|_{p}
$$

for all $x \in[a, b]$, where

$$
B(x, q)=\left[\left(\frac{(x-a)^{q+1}}{q+1}\right)^{\frac{1}{q}}+\frac{1}{2}\left(\frac{(b-a)^{q+1}}{2 q+1}\right)^{\frac{1}{q}}+\frac{1}{2}(b-a)(x-a)^{\frac{1}{q}}\right]
$$

and $\frac{1}{p}+\frac{1}{q}=1$.

Proof. Clearly, by Theorem 1.1 one can easily find that

$$
f(x)=f(a)+(x-a) f^{\prime}(a)+\int_{a}^{x}(x-t) f^{\prime \prime}(t) d t .
$$

Similarly

$$
\begin{aligned}
\frac{1}{b-a} \int_{a}^{b} f(t) d t & =\frac{1}{b-a}(F(b)-F(a)) \\
& =\frac{1}{b-a}\left((b-a) F^{\prime}(a)+\frac{1}{2}(b-a)^{2} F^{\prime \prime}(a)+\int_{a}^{b} \frac{(b-x)^{2}}{2} F^{\prime \prime \prime}(t) d t\right) \\
& =f(a)+\frac{1}{2}(b-a) f^{\prime}(a)+\frac{1}{b-a} \int_{a}^{b} \frac{(b-x)^{2}}{2} f^{\prime \prime}(t) d t .
\end{aligned}
$$

On the other hand by Fundamental Theorem of Calculus,

$$
f^{\prime}(x)=f^{\prime}(a)+\int_{a}^{x} f^{\prime \prime}(t) d t .
$$


By multiplying the appropriate coefficients of the above equalities and adding the resulting equality, we find that

$$
\begin{aligned}
& f(x)-\frac{1}{b-a} \int_{a}^{b} f(t) d t-\left(x-\frac{a+b}{2}\right) f^{\prime}(x) \\
= & \int_{a}^{x}(x-t) f^{\prime \prime}(t) d t-\frac{1}{b-a} \int_{a}^{b} \frac{(b-x)^{2}}{2} f^{\prime \prime}(t) d t-\left(x-\frac{a+b}{2}\right) \int_{a}^{x} f^{\prime \prime}(t) d t .
\end{aligned}
$$

Taking modulus of (2.14), we get

$$
\begin{aligned}
& \quad\left|f(x)-\frac{1}{b-a} \int_{a}^{b} f(t) d t-\left(x-\frac{a+b}{2}\right) f^{\prime}(x)\right| \\
& \leq\left|\int_{a}^{x}(x-t) f^{\prime \prime}(t) d t\right|+\frac{1}{b-a}\left|\int_{a}^{b} \frac{(b-x)^{2}}{2} f^{\prime \prime}(t) d t\right|+\left|\left(x-\frac{a+b}{2}\right) \int_{a}^{x} f^{\prime \prime}(t) d t\right| .
\end{aligned}
$$

Then, by the well-known Hölder inequality, we obtain

$$
\left|\int_{a}^{b} \frac{(b-x)^{2}}{2} f^{\prime \prime}(t) d t\right| \leq \frac{1}{2}\left\|f^{\prime \prime}\right\|_{p}\left(\int_{a}^{b}(b-x)^{2 q} d t\right)^{\frac{1}{q}}=\frac{1}{2}\left\|f^{\prime \prime}\right\|_{p}\left(\frac{(b-a)^{2 q+1}}{2 q+1}\right)^{\frac{1}{q}}
$$

and

$$
\begin{aligned}
\left|\left(x-\frac{a+b}{2}\right) \int_{a}^{x} f^{\prime \prime}(t) d t\right| & \leq \frac{b-a}{2}\left|\int_{a}^{x} f^{\prime \prime}(t) d t\right| \\
& \leq \frac{b-a}{2}\left\|f^{\prime \prime}\right\|_{p}\left(\int_{a}^{x} 1^{q} d t\right)^{\frac{1}{q}} \\
& =\frac{b-a}{2}(x-a)^{\frac{1}{q}}\left\|f^{\prime \prime}\right\|_{p} .
\end{aligned}
$$

If we substitute (2.11), (2.16) and (2.17) into (2.15), we have

$$
\begin{aligned}
& \left|f(x)-\frac{1}{b-a} \int_{a}^{b} f(t) d t-\left(x-\frac{a+b}{2}\right) f^{\prime}(x)\right| \\
\leq & \left\|f^{\prime \prime}\right\|_{p}\left[\left(\frac{(x-a)^{q+1}}{q+1}\right)^{\frac{1}{q}}+\frac{1}{2}\left(\frac{(b-a)^{q+1}}{2 q+1}\right)^{\frac{1}{q}}+\frac{1}{2}(b-a)(x-a)^{\frac{1}{q}}\right] .
\end{aligned}
$$

This completes the proof.

2.4. Corollary. Under the assumptions of Theorem 2.1, if we choose $x=\frac{a+b}{2}$, then we have the following midpoint type inequality

$$
\begin{aligned}
\left|f\left(\frac{a+b}{2}\right)-\frac{1}{b-a} \int_{a}^{b} f(t) d t\right| & \leq\left\|f^{\prime \prime}\right\|_{p}\left(\frac{b-a}{2}\right)^{1+\frac{1}{q}} \\
& \times\left[\left(\frac{1}{q+1}\right)^{\frac{1}{q}}+\left(\frac{2}{2 q+1}\right)^{\frac{1}{q}}+1\right] .
\end{aligned}
$$


2.5. Corollary. Under the same assumptions of Theorem 2.3 with $x=a$ and $x=b$, respectively, then adding the resulting inequalities and using the property triangle inequality for the modulus, we get the following inequality

$$
\begin{aligned}
& \left|\frac{f(a)+f(b)}{2}-\frac{1}{b-a} \int_{a}^{b} f(t) d t-\frac{b-a}{4}\left[f^{\prime}(b)-f^{\prime}(a)\right]\right| \\
& \leq \frac{1}{2}\left\|f^{\prime \prime}\right\|_{p}(b-a)^{1+\frac{1}{q}}\left[\left(\frac{1}{q+1}\right)^{\frac{1}{q}}+\left(\frac{1}{2 q+1}\right)^{\frac{1}{q}}+\frac{1}{2}\right] .
\end{aligned}
$$

2.6. Theorem. Let $f:[a, b] \rightarrow \mathbb{R}$ be a twice continuously differentiable mapping on $(a, b)$ with $f^{\prime \prime} \in L^{p}(a, b), 1 \leqq p<\infty$, we have

$$
\begin{aligned}
& \mid f(x)-\left(x-\frac{a+b}{2}\right) f^{\prime}(x)-\frac{1}{b-a} \int_{a}^{b} f(t) d t \\
+ & {\left[\frac{1}{24}(b-a)^{2}+\frac{1}{2}\left(x-\frac{a+b}{2}\right)^{2}\right] \frac{f^{\prime}(b)-f^{\prime}(a)}{b-a} \mid } \\
\leq & \left\|f^{\prime \prime}\right\|_{p} C(x, q)
\end{aligned}
$$

where

$$
\begin{aligned}
C(x, q) & =\left[\left(\frac{(x-a)^{q+1}}{q+1}\right)^{\frac{1}{q}}+\frac{b-a}{2}(x-a)^{\frac{1}{q}}\right. \\
& \left.+\frac{1}{2(b-a)}\left(\frac{(b-a)^{2 q+1}-(b-x)^{2 q+1}}{2 q+1}\right)^{\frac{1}{q}}+\frac{(b-a)^{1+\frac{1}{q}}}{6}\right]
\end{aligned}
$$

and $\frac{1}{p}+\frac{1}{q}=1$.

Proof. By Fundamental Theorem of Calculus, we have

$$
f^{\prime}(b)-f^{\prime}(a)=\int_{a}^{b} f^{\prime \prime}(t) d t .
$$

Then combining equalities (2.4), (2.12), (2.13) and (2.19), we deduce that

$$
\begin{aligned}
& f(x)-\left(x-\frac{a+b}{2}\right) f^{\prime}(x)-\frac{1}{b-a} \int_{a}^{b} f(t) d t \\
+ & {\left[\frac{1}{24}(b-a)^{2}+\frac{1}{2}\left(x-\frac{a+b}{2}\right)^{2}\right] \frac{f^{\prime}(b)-f^{\prime}(a)}{b-a} } \\
= & \int_{a}^{x}(x-t) f^{\prime \prime}(t) d t-\left(x-\frac{a+b}{2}\right) \int_{a}^{x} f^{\prime \prime}(t) d t-\frac{1}{2(b-a)} \int_{a}^{x}(b-t)^{2} f^{\prime \prime}(t) d t \\
+ & \frac{1}{b-a}\left[\frac{1}{24}(b-a)^{2}+\frac{1}{2}\left(x-\frac{a+b}{2}\right)^{2}\right] \int_{a}^{b} f^{\prime \prime}(t) d t .
\end{aligned}
$$


To end the proof, it remains to find an upper bound of equality (2.20). Therefore using the properties of modulus, we have

$$
\begin{aligned}
& \quad \mid f(x)-\left(x-\frac{a+b}{2}\right) f^{\prime}(x)-\frac{1}{b-a} \int_{a}^{b} f(t) d t \\
& +\left[\frac{1}{24}(b-a)^{2}+\frac{1}{2}\left(x-\frac{a+b}{2}\right)^{2}\right] \frac{f^{\prime}(b)-f^{\prime}(a)}{b-a} \mid \\
& \leq\left|\int_{a}^{x}(x-t) f^{\prime \prime}(t) d t\right|+\left|\left(x-\frac{a+b}{2}\right) \int_{a}^{x} f^{\prime \prime}(t) d t\right| \\
& +\quad \frac{1}{2(b-a)}\left|\int_{a}^{x}(b-t)^{2} f^{\prime \prime}(t) d t\right| \\
& +\quad \frac{1}{b-a}\left|\left[\frac{1}{24}(b-a)^{2}+\frac{1}{2}\left(x-\frac{a+b}{2}\right)^{2}\right] \int_{a}^{b} f^{\prime \prime}(t) d t\right| .
\end{aligned}
$$

Then, by the well-known Hölder inequality, one finds that

$$
\begin{aligned}
\left|\int_{a}^{x}(b-t)^{2} f^{\prime \prime}(t) d t\right| & \leq\left\|f^{\prime \prime}\right\|_{p}\left(\int_{a}^{x}(b-t)^{2 q} d t\right)^{\frac{1}{q}} \\
& =\left\|f^{\prime \prime}\right\|_{p}\left(\frac{(b-a)^{2 q+1}-(b-x)^{2 q+1}}{2 q+1}\right)^{\frac{1}{q}}
\end{aligned}
$$

and

$$
\begin{aligned}
& \frac{1}{b-a}\left|\left[\frac{1}{24}(b-a)^{2}+\frac{1}{2}\left(x-\frac{a+b}{2}\right)^{2}\right] \int_{a}^{b} f^{\prime \prime}(t) d t\right| \\
\leq & \frac{b-a}{6}\left|\int_{a}^{b} f^{\prime \prime}(t) d t\right| \leq \frac{b-a}{6}\left\|f^{\prime \prime}\right\|_{p}\left(\int_{a}^{b} 1^{q} d t\right)^{\frac{1}{q}} \\
= & \frac{(b-a)^{1+\frac{1}{q}}}{6}\left\|f^{\prime \prime}\right\|_{p} .
\end{aligned}
$$


If we substitute (2.11), (2.17), (2.22) and (2.23) into (2.21), we have

$$
\begin{aligned}
& \mid f(x)-\left(x-\frac{a+b}{2}\right) f^{\prime}(x)-\frac{1}{b-a} \int_{a}^{b} f(t) d t \\
& +\left[\frac{1}{24}(b-a)^{2}+\frac{1}{2}\left(x-\frac{a+b}{2}\right)^{2}\right] \frac{f^{\prime}(b)-f^{\prime}(a)}{b-a} \mid \\
& \leq\left\|f^{\prime \prime}\right\|_{p}\left[\left(\frac{(x-a)^{q+1}}{q+1}\right)^{\frac{1}{q}}+\frac{b-a}{2}(x-a)^{\frac{1}{q}}\right. \\
& \left.+\frac{1}{2(b-a)}\left(\frac{(b-a)^{2 q+1}-(b-x)^{2 q+1}}{2 q+1}\right)^{\frac{1}{q}}+\frac{(b-a)^{1+\frac{1}{q}}}{6}\right]
\end{aligned}
$$

which completes the proof.

2.7. Corollary. Under the assumptions of Theorem 2.1 with $x=\frac{a+b}{2}$, we have the following inequality

$$
\begin{aligned}
& \left|f\left(\frac{a+b}{2}\right)+\frac{1}{24}(b-a)\left[f^{\prime}(b)-f^{\prime}(a)\right]-\frac{1}{b-a} \int_{a}^{b} f(t) d t\right| \\
\leq & \left\|f^{\prime \prime}\right\|_{p}\left(\frac{b-a}{2}\right)^{1+\frac{1}{q}}\left[\left(\frac{1}{q+1}\right)^{\frac{1}{q}}+\frac{1}{4}\left(\frac{2^{2 q+1}-1}{2 q+1}\right)^{\frac{1}{q}}+\frac{3+2^{\frac{1}{q}}}{3}\right] .
\end{aligned}
$$

2.8. Corollary. Under the same assumptions of Theorem 2.6 with $x=a$ and $x=b$, respectively, then adding the resulting inequalities and using the property triangle inequality for the modulus, we get the following inequality

$$
\begin{aligned}
& \mid \frac{f(a)+f(b)}{2}-\frac{b-a}{12}\left[f^{\prime}(b)-f^{\prime}(a)\right]-\frac{1}{b-a} \int_{a}^{b} f(t) d t \\
\leq & \left\|f^{\prime \prime}\right\|_{p} \frac{(b-a)^{1+\frac{1}{q}}}{2}\left[\left(\frac{1}{q+1}\right)^{\frac{1}{q}}+\frac{1}{2}\left(\frac{1}{2 q+1}\right)^{\frac{1}{q}}+\frac{5}{6}\right] .
\end{aligned}
$$

\section{Numerical Experiments}

We now deal with applications of the integral inequalities to obtain estimates of composite quadrature rules.

Consider the partition of the interval $[a, b]$, given by

$$
I_{n}: a=x_{0}<x_{1}<\ldots<x_{n-1}<x_{n}=b,
$$

such that $h_{i}=x_{i+1}-x_{i}=\frac{b-a}{n}, i=0, \ldots, n-1$.

3.1. Experiment 1. We obtain the following Theorem by using Corollary 2.2-1.

3.1. Theorem. The assumptions of Theorem 2.1 hold. Then we have the representation

$$
\frac{1}{b-a} \int_{a}^{b} f(t) d t=S_{T}\left(f, I_{n}\right)+R_{T}\left(f, I_{n}\right)
$$


where $S_{T}\left(f, I_{n}\right)$ is as defined by

$$
S_{T}\left(f, I_{n}\right):=\frac{1}{2 n} \sum_{i=0}^{n-1}\left[f\left(x_{i}\right)+f\left(x_{i+1}\right)\right],
$$

and the remainder term $R_{T}\left(f, I_{n}\right)$ satisfies the estimation:

$$
\left|R_{T}\left(f, I_{n}\right)\right| \leq \frac{1}{n^{2}} \frac{(b-a)^{1+\frac{1}{q}}}{2(2 q+1)^{\frac{1}{q}}}\left\|f^{\prime \prime}\right\|_{p} .
$$

Proof. Applying Corollary 2.2 on the interval $\left[x_{i}, x_{i+1}\right]$, we obtain

$$
\left.\left|\frac{n}{b-a} \int_{x_{i}}^{x_{i+1}} f(t) d t-\frac{f\left(x_{i}\right)+f\left(x_{i+1}\right)}{2}\right| \leq \frac{\left(\frac{b-a}{n}\right)^{1+\frac{1}{q}}}{2(2 q+1)^{\frac{1}{q}}}\left\|f^{\prime \prime}\right\|_{p,\left[x_{i}, x_{i+1}\right.}\right]
$$

for all $i=0, \ldots, n-1$. Summing over $i$ from 0 to $n-1$ and using the triangle inequality we obtain

$$
\left|\frac{n}{b-a} \int_{a}^{b} f(t) d t-\frac{1}{2} \sum_{i=0}^{n-1}\left[f\left(x_{1}\right)+f\left(x_{i+1}\right)\right]\right| \leq \frac{\left(\frac{b-a}{n}\right)^{1+\frac{1}{q}}}{2(2 q+1)^{\frac{1}{q}}} \sum_{i=0}^{n-1}\left\|f^{\prime \prime}\right\|_{p,\left[x_{i}, x_{i+1}\right]} .
$$

Define

$$
\xi_{i}=\int_{x_{i}}^{x_{i+1}}\left|f^{\prime}(t)\right|^{p} d t
$$

then using the discrete Hölder inequality, we have

$$
\sum_{i=0}^{n-1}\left\|f^{\prime \prime}\right\|_{p,\left[x_{i}, x_{i+1}\right]}=\sum_{i=0}^{n-1}\left(\xi_{i}\right)^{\frac{1}{p}} \leq n^{1-\frac{1}{p}}\left(\sum_{i=0}^{n-1} \xi_{i}\right)^{\frac{1}{p}}=n^{1-\frac{1}{p}}\left\|f^{\prime \prime}\right\|_{p} .
$$

Thus we have

$$
\left|R_{T}\left(f, I_{n}\right)\right| \leq \frac{1}{n^{2}} \frac{(b-a)^{1+\frac{1}{q}}}{2(2 q+1)^{\frac{1}{q}}}\left\|f^{\prime \prime}\right\|_{p} .
$$

Hence, the proof is completed.

3.2. Experiment 2. We obtain the following Theorem by using Corollary 2.4.

3.2. Theorem. The assumptions of Theorem 2.3 hold. Then we have the representation

$$
\frac{1}{b-a} \int_{a}^{b} f(t) d t=S_{M}\left(f, I_{n}\right)+R_{M}\left(f, I_{n}\right)
$$

where $S_{M}\left(f, I_{n}\right)$ is as defined by

$$
S_{M}\left(f, I_{n}\right):=\frac{1}{n} \sum_{i=0}^{n-1} f\left(\frac{x_{i}+x_{i+1}}{2}\right),
$$

and the remainder term $R_{M}\left(f, I_{n}\right)$ satisfies the estimation:

$$
\left|R_{M}\left(f, I_{n}\right)\right| \leq \frac{1}{n^{2}}\left(\frac{b-a}{2}\right)^{1+\frac{1}{q}}\left[\left(\frac{1}{q+1}\right)^{\frac{1}{q}}+\left(\frac{2}{2 q+1}\right)^{\frac{1}{q}}+1\right]\left\|f^{\prime \prime}\right\|_{p} .
$$


Proof. Applying Corollary 2.4 on the interval $\left[x_{i}, x_{i+1}\right]$, we obtain

$$
\begin{aligned}
\left|f\left(\frac{x_{i}+x_{i+1}}{2}\right)-\frac{n}{b-a} \int_{x_{i}}^{x_{i+1}} f(t) d t\right| & \leq\left\|f^{\prime \prime}\right\|_{p,\left[x_{i}, x_{i+1}\right]}\left(\frac{b-a}{2 n}\right)^{1+\frac{1}{q}} \\
& \times\left[\left(\frac{1}{q+1}\right)^{\frac{1}{q}}+\left(\frac{2}{2 q+1}\right)^{\frac{1}{q}}+1\right] .
\end{aligned}
$$

for all $i=0, \ldots, n-1$. Summing over $i$ from 0 to $n-1$ and using the triangle inequality we obtain

$$
\begin{aligned}
\left|\sum_{i=0}^{n-1} f\left(\frac{x_{i}+x_{i+1}}{2}\right)-\frac{n}{b-a} \int_{a}^{b} f(t) d t\right| & \leq\left(\frac{b-a}{2 n}\right)^{1+\frac{1}{q}} \\
& \times\left[\left(\frac{1}{q+1}\right)^{\frac{1}{q}}+\left(\frac{2}{2 q+1}\right)^{\frac{1}{q}}+1\right] \\
& \times \sum_{i=0}^{n-1}\left\|f^{\prime \prime}\right\|_{p,\left[x_{i}, x_{i+1}\right]} .
\end{aligned}
$$

Similarly using the discrete Hölder inequality, we have

$$
\left|R_{M}\left(f, I_{n}\right)\right| \leq \frac{1}{n^{2}}\left(\frac{b-a}{2}\right)^{1+\frac{1}{q}}\left[\left(\frac{1}{q+1}\right)^{\frac{1}{q}}+\left(\frac{2}{2 q+1}\right)^{\frac{1}{q}}+1\right]\left\|f^{\prime \prime}\right\|_{p}
$$

which completes the proof.

\section{Concluding Remarks}

In this study, new bounds of Ostrowski type integral inequalities have been presented and tested. Some numerical examples have been given for validation.

\section{References}

[1] G. A. Anastassiou and S. S. Dragomir, On some estimates of the remainder in Taylor's formula, J. Math. Anal. Appl. 263 (2001), no. 1, 246-263.

[2] P. Cerone, S. S. Dragomir, J. Roumeliotis, An inequality of Ostrowski type for mappings whose second derivatives are bounded and applications, RGMIA Res. Rep. Coll., 1 (1998).

[3] X.-L. Cheng. Improvement of some Ostrowski-Grüss type inequalities, Computers \& Mathematics with Applications, 42, 109-114, 2001.

[4] S. S. Dragomir, S. Wang, An inequality of Ostrowski-Grüss type and its applications to the estimation of error bounds for some special means and for some numerical quadrature rules, Comput. Math. Appl., 33 (1997), 15-20.

[5] S. S. Dragomir and S. Wang, An inequality of Ostrowski-Gruss type and its applications to the estimation of error bounds for some special means and for some numerical quadrature rules, Comput. Math. Appl. 33 (1997), no. 11, 15-20.

[6] V. N. Huy and Q. A. Ngo, New bounds for the Ostrowski-like type inequalities, Bull. Korean Math. Soc. 48 (2011) 95-104.

[7] A. R. Kashif, , M. Shoaib, M. A. Latif, Improved version of perturbed Ostrowski type inequalities for $n$-times differentiable mappings with three-step kernel and its application, J. Nonlinear Sci. Appl. 9 (2016), 3319-3332.

[8] M. E. Kiris and M. Z. Sarikaya, On Ostrowski type inequalities and Cebyšev type inequalities, Filomat, 29:8 (2015), 1695-1713.

[9] Z. Liu, Some Ostrowski-Grüss type inequalities and applications, Comput. Math. Appl. 53 (2007) 73-79.

[10] Z. Liu, Some Ostrowski type inequalities, Math. Comput. Modelling 48 (2008) 949-960. 
[11] W. Liu, New bounds for the companion of Ostrowski's inequality and applications, Filomat, 28 (2014), 167-178.

[12] A. M. Ostrowski, Über die absolutabweichung einer differentiebaren funktion von ihrem integralmitelwert, Comment. Math. Helv. 10(1938), 226-227.

[13] M. Z. Sarikaya and H. Yaldiz, New generalization fractional inequalities of Ostrowski-Grüss type, Lobachevskii Journal of Math., 2013, Vol. 34, No. 4, pp. 326-331.

[14] A. Qayyum, S. Hussain, A new generalized Ostrowski Gr "uss type inequality and applications, Appl. Math. Lett., 25 (2012),

[15] 1875-1880.

[16] N. Ujević, New bounds for the first inequality of Ostrowski-Grüss type and applications, Comput. Math. Appl., 46 (2003), 421-427. 